\title{
Evidence of oxygen-dependent modulation in $\mathrm{LuFe}_{2} \mathrm{O}_{4}$
}

\author{
J. Bourgeois, ${ }^{1,2, *}$ M. Hervieu, ${ }^{1}$ M. Poienar, ${ }^{3}$ A. M. Abakumov, ${ }^{4}$ E. Elkaïm, ${ }^{5}$ M. T. Sougrati, ${ }^{3}$ F. Porcher,${ }^{2}$ F. Damay, ${ }^{2}$ \\ J. Rouquette, ${ }^{3}$ G. Van Tendeloo, ${ }^{4}$ A. Maignan, ${ }^{1}$ J. Haines, ${ }^{3}$ and C. Martin ${ }^{1}$ \\ ${ }^{1}$ Laboratoire CRISMAT, ENSICAEN, UMR 6508 CNRS, 6 Boulevard du Maréchal Juin, 14050 Caen Cedex, France \\ ${ }^{2}$ Laboratoire Léon Brillouin, UMR 12, CEA-Saclay, CEA-CNRS, 91191 Gif-sur-Yvette Cedex, France \\ ${ }^{3}$ Institut Charles Gerhardt UMR CNRS 5253, Université Montpellier II, Place Eugène Bataillon, cc1503, 34095 Montpellier Cedex 5, France \\ ${ }^{4}$ EMAT, University of Antwerp, Groenenborgerlaan 171, B-2020 Antwerpen, Belgium \\ ${ }^{5}$ Synchrotron Soleil, L'Orme des Merisiers, Saint-Aubin BP 4891192 Gif-sur-Yvette Cedex, France
}

(Received 27 October 2011; published 6 February 2012)

\begin{abstract}
A polycrystalline sample of $\mathrm{LuFe}_{2} \mathrm{O}_{4}$ has been investigated by means of powder synchrotron $\mathrm{x}$-ray and neutron diffraction and transmission electron microscopy (TEM), along with Mössbauer spectroscopy and transport and magnetic properties. A monoclinic distortion is unambiguously evidenced, and the crystal structure is refined in the monoclinic $C 2 / m$ space group $\left[a_{M}=5.9563(1) \AA, b_{M}=3.4372(1) \AA, c_{M}=8.6431(1) \AA, \beta=103.24(1)^{\circ}\right]$. Along with the previously reported modulations distinctive of the charge-ordering (CO) of the iron species, a new type of incommensurate order is observed, characterized by a vector $\vec{q}_{1}=\alpha_{1} \overrightarrow{\mathrm{a}}_{M}^{*}+\gamma_{1} \vec{c}_{M}^{*}$ (with $\alpha_{1} \cong 0.55$, $\gamma_{1} \cong 0.13$ ). In situ heating TEM observations from 300 to $773 \mathrm{~K}$ confirm that the satellites associated with $\vec{q}_{1}$ vanish completely, only at a temperature significantly higher than the $\mathrm{CO}$ temperature. This incommensurate modulation has a displacive character and corresponds primarily to a transverse displacive modulation wave of the Lu cations position, as revealed by the high resolution, high angle annular dark field scanning TEM images and in agreement with synchrotron data refinements. Analyses of vacuum-annealed samples converge toward the hypothesis of a new ordering mechanism, associated with a tiny oxygen deviation from the $\mathrm{O}_{4}$ stoichiometry.
\end{abstract}

\section{INTRODUCTION}

Owing to their great technological and fundamental importance, enormous progress has been made in the field of multiferroic materials, i.e., materials that show the coexistence and a strong interplay between two $a$ priori unrelated phenomena: ferroelectricity and magnetism. ${ }^{1-3}$ Among them, charge-ordered transition metal oxides in which the electronic ferroelectricity is determined by the charge ordering (CO) and thus by electron correlations and/or electron-lattice interactions ${ }^{4,5}$ constitute a special class in which $\mathrm{LuFe}_{2} \mathrm{O}_{4}$ is often cited as the prototype. $\mathrm{LuFe}_{2} \mathrm{O}_{4}$ belongs to the $\mathrm{RFe}_{2} \mathrm{O}_{4}$ family where $\mathrm{R}$ is a rare-earth cation from Dy to $\mathrm{Lu}$ or $\mathrm{Y}^{6}$ These compounds crystallize at room temperature (RT) in the $R \overline{3} m$ space group and exhibit a layered structure (2D), described as the 1:1 stacking along the $c$ axis of $\left[\mathrm{LuO}_{2}\right]_{\infty}$ and $\left[\mathrm{Fe}_{2} \mathrm{O}_{4}\right]_{\infty}$ bilayers. $^{7}$

The average iron valence in this compound is +2.5 , and the $\mathrm{Fe}^{2+} / \mathrm{Fe}^{3+} \mathrm{CO}$ that occurs at $T_{\mathrm{CO}} \cong 330 \mathrm{~K}$ induces ferroelectricity with a quite large value of the ferroelectric polarization, i.e., about $26 \mu \mathrm{C} \mathrm{cm}^{-2}$. ${ }^{8}$ The pyroelectric signal shows an unusual step around $250 \mathrm{~K}$, which is very close to the spin-ordering temperature of $240 \mathrm{~K} .{ }^{9}$ Moreover, multiple charge-ordered phase transitions are reported, i.e., 3D charge order below $330 \mathrm{~K}$ followed by 2D charge correlations up to $500 \mathrm{~K}^{10,11}$

The magnetic behavior of $\mathrm{LuFe}_{2} \mathrm{O}_{4}$ is rather complex; strong discrepancies emerge from the literature with regards to the number of transitions and the appropriate magnetic models: ferrimagnetic clusters below $T_{\mathrm{N}}=240 \mathrm{~K}^{9}$ but also two 3D magnetic transitions at $T_{\mathrm{N}}=240 \mathrm{~K}$ and $T_{\mathrm{L}}=175 \mathrm{~K} .{ }^{10}$ Numerous reports also mention the spin-glass character of this compound at low temperature ${ }^{9,12,13}$ whose origin is unclear, even if a parallel can be made with the Verwey transition in
$\mathrm{Fe}_{3} \mathrm{O}_{4} \cdot{ }^{14}$ The situation is also complex because of aging and memory effects which appear also as important parameters, probably in connection with the spin-glass state. ${ }^{13}$

Similarly, the CO state remains till now somewhat controversial and moreover seems to be sample and temperature dependent. ${ }^{10,11,15-17}$ For example, a $\sqrt{3} \times \sqrt{3}$ supercell in the bilayer planes with two distinct structural modulations, a major $\vec{q}_{1}=[1 / 3,1 / 3,2]$ and a weak $\vec{q}_{2}=\vec{q}_{1} / 10+[0,0,3 / 2]$, has been proposed ${ }^{15}$ but also a simpler model with only $\mathrm{Fe}^{2+}$ and $\mathrm{Fe}^{3+}$ in alternate layers. ${ }^{18}$

These results have also to be compared with those obtained for $\mathrm{YFe}_{2} \mathrm{O}_{4}$, belonging to the same class of oxides, for which structural transitions were observed vs temperature. In that case, a strong influence of the oxygen content is evidenced on the charge-ordered structure and on the physical properties, ${ }^{19-23}$ contrary to $\mathrm{LuFe}_{2} \mathrm{O}_{4}$, which was believed to be free from oxygen nonstoichiometry effects. ${ }^{24}$ However, the discrepancies reported in the literature call for a thorough investigation of a unique sample, which is the focus of this article. Complementary techniques like electron, neutron, and synchrotron X-ray diffraction, as well as Mössbauer and magnetic characterizations, were performed to obtain a precise description of the RT state of $\mathrm{LuFe}_{2} \mathrm{O}_{4}$. The main feature revealed by this study is the evidence of a new incommensurate order that could be attributed to a very small excess of oxygen.

\section{EXPERIMENTAL}

A $10 \mathrm{~g}$ polycrystalline $\mathrm{LuFe}_{2} \mathrm{O}_{4}$ sample was prepared via solid-state reaction. A mixture of $\mathrm{Lu}_{2} \mathrm{O}_{3}, \mathrm{Fe}_{2} \mathrm{O}_{3}$, and $\mathrm{Fe}$ was ground in an agate mortar before being pressed into a rod of $6 \mathrm{~mm}$ diameter and about $10 \mathrm{~cm}$ length. The sample was 
then sealed in an evacuated silica tube and heated up to $1180^{\circ} \mathrm{C}$ for $12 \mathrm{hrs}$. It was then checked by RT x-ray powder diffraction.

Magnetic susceptibility (defined as $\chi=M / H$ ) was calculated from magnetization data measured in a magnetic field of 100 Oe, in zero field cooling (ZFC) and field cooling (FC) modes, and on warming from 1.5 to $400 \mathrm{~K}$ using a Quantum Design SQUID magnetometer. Resistivity measurements vs temperature were carried out using a four-point technique in a Physical Property Measurement System (PPMS, Quantum Design $)$ on a small bar $\left(d=1.23 \mathrm{~mm}, S=1.82 \times 1.32 \mathrm{~mm}^{2}\right)$ cut in the rod.

The RT synchrotron X-ray powder diffraction experiment was carried out on the CRISTAL beamline at SOLEIL, using a wavelength $\lambda=0.56356 \AA$. A small amount of sample, crushed and sifted at $63 \mu \mathrm{m}$, was put in a $0.3 \mathrm{~mm}$ quartz capillary. High resolution neutron powder diffraction (NPD) data were recorded at 300 and $350 \mathrm{~K}$ on the $3 \mathrm{~T} 2$ diffractometer $(\lambda=$ $1.2253 \AA$ ) located at LLB-Orphée; the rod about $10 \mathrm{~g}$ was put in a vanadium can. To analyze these diffraction data, Rietveld refinements were performed with the programs of the FullProf suite, ${ }^{25}$ and the JANA2006 software was used to go further in the study of the structural modulations. ${ }^{26}$

${ }^{57} \mathrm{Fe}$ Mössbauer spectra were recorded in transmission geometry on a standard EG\&G spectrometer in the constant acceleration mode using ${ }^{57} \mathrm{Co}(\mathrm{Rh})$. All isomer shifts are given with respect to the RT spectrum of $\alpha-\mathrm{Fe}$.

Transmission electron microscopy (TEM) specimens were prepared by crushing powder samples under ethanol in an agate mortar and depositing the drops onto a holey carbon grid. Electron diffraction (ED) observations at RT were carried out using a JEOL $200 \mathrm{CX}$ microscope equipped with a tilt-rotation sample holder $\left( \pm 60^{\circ}\right)$ and the high-resolution electron microscopy (HREM) with a TOPCON microscope (operating at $200 \mathrm{kV}$ with an aberration constant $\mathrm{Cs}=0.4 \mathrm{~mm}$ ). ED patterns in the $300-880 \mathrm{~K}$ temperature range were taken with a Tecnai G2 electron microscope operated at $200 \mathrm{kV}$ and equipped with a GATAN heating holder. High resolution, high angle annular dark field scanning TEM (HAADF-STEM) images were obtained using a Titan G3 electron microscope operated at $300 \mathrm{kV}$ and equipped with the probe aberration corrector.

\section{RESULTS}

\section{A. Preliminary characterizations}

The RT x-ray diffraction pattern is fully indexed in the

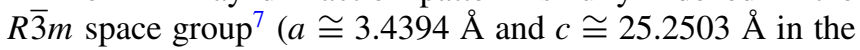
hexagonal setting); it shows that the sample is a single-phase and well-crystallized. The $\mathrm{LuFe}_{2} \mathrm{O}_{4}$ ferrite structure (Fig. 1) can be described as a stacking along $c$ of $\left[\mathrm{LuO}_{2}\right]_{\infty}$ and $\left[\mathrm{Fe}_{2} \mathrm{O}_{4}\right]_{\infty}$ layers (with two common $[\mathrm{O}]_{\infty}$ sheets). Lutetium cations on the $3 a\left(\begin{array}{lll}0 & 0 & 0\end{array}\right)$ site are coordinated by six oxygen ions $\mathrm{O}(1)$ in the $6 c(00 \mathrm{z} \cong 0.2882)$ site forming edge-sharing octahedra, leading to a $\mathrm{CdI}_{2}$-type sheet. The iron cations on the $6 c$ site $(00 z \cong 0.2148)$ are coordinated by five oxygen ions: one $\mathrm{O}(1)$ and four $\mathrm{O}(2)$ —also in the $6 c(00 \mathrm{z} \cong 0.1311)$ site-forming a trigonal bipyramid. Fe cations are actually not located at the center of these bipyramids but displaced toward (a)

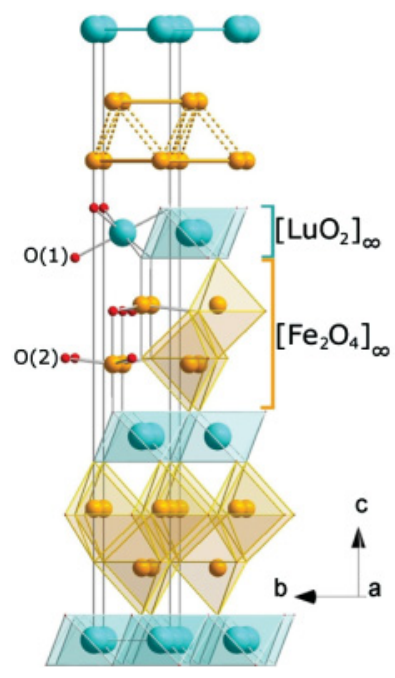

(b)

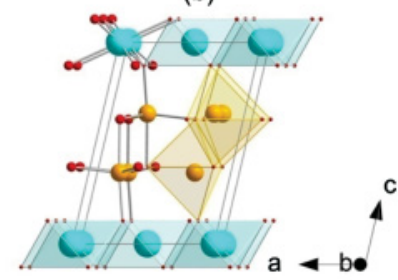

FIG. 1. (Color online) Structure of $\mathrm{LuFe}_{2} \mathrm{O}_{4}$ described in (a) $R \overline{3} m$ and (b) $C 2 / m$. The Fe-Fe distances in the $\mathrm{W}$ layer are shown [in (a)] with continuous and dotted lines, differentiating the in- and out-ofplane distances, respectively.

the $\left[\mathrm{LuO}_{2}\right]_{\infty}$ layers, with two unequal Fe-O(1) distances along the $c$-axis. These bipyramids share corners in the $(a, b)$ plane and share edges in the third direction to form the $\left[\mathrm{Fe}_{2} \mathrm{O}_{4}\right]_{\infty}$ bilayers. Each iron layer forms a perfect triangular array, and a bilayer can be described as two layers separated from $\left(2 z_{\mathrm{Fe}}-1 / 3\right)$ along $c$ and shifted by the [1/3 2/3 0] vector in the basal plane. The iron bilayer can also be described as a buckled honeycomb lattice, with a unique characteristic $\mathrm{Fe}-\mathrm{Fe}$ interatomic distance, which is the shortest of the Fe-Fe distances [dotted lines in Fig. 1(a)].

The magnetic susceptibility vs temperature curve, reported in Fig. 2, is similar to those reported for this compound. ${ }^{27,28} \mathrm{It}$ is characteristic of antiferromagnetism with a Néel temperature of about $250 \mathrm{~K}$; the origin of the broader transitions that can be seen at lower temperature is unclear up to now. The large difference that exists between the ZFC and FC modes is also a known characteristic of this type of compounds and is still a matter of debate. A more detailed investigation of magnetic properties and magnetic structures studied by

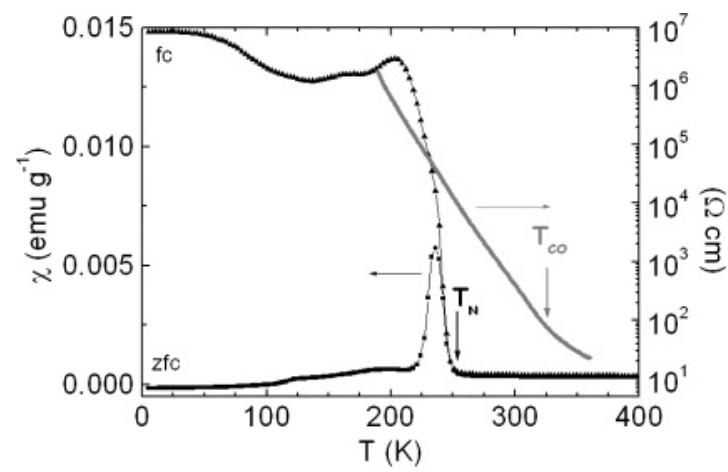

FIG. 2. Evolution with temperature of the ZFC and FC magnetization recorded in 100 Oe (left $y$-axis) and of the resistivity (right $y$-axis). The $\mathrm{CO}$ and magnetic ordering temperatures $\left(T_{\mathrm{CO}}, T_{\mathrm{N}}\right)$ are evidenced by arrows. 


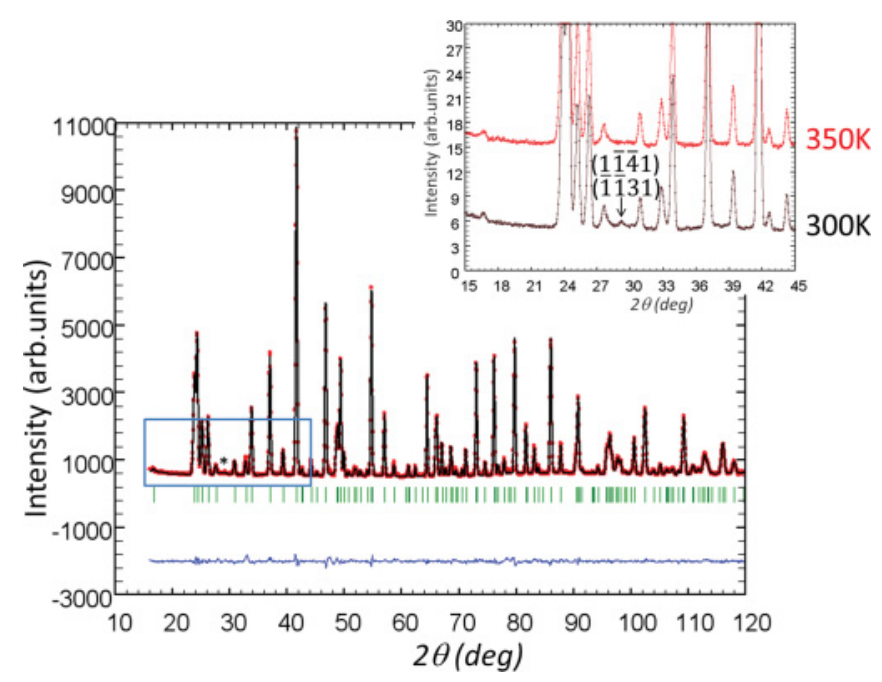

FIG. 3. (Color online) Rietveld refinement of the $300 \mathrm{~K}$ neutron diffraction data (experimental data: open circles; calculated profile: continuous line; allowed Bragg reflections: vertical marks. The difference between the experimental and calculated profiles is displayed at the bottom of the graph.) Inset: Enlargement of the low-angle part of the pattern illustrating the disappearance between 300 and $350 \mathrm{~K}$ of the $\mathrm{CO}$ peak (indicated by a star in the main panel) indexed by using equivalent propagation vector of $\vec{q} 2$ in $R \overline{3} m$.

neutron diffraction will be reported separately. ${ }^{29}$ The resistivity curve, also given in Fig. 2, is shown for a limited temperature range, the sample becoming too insulating for the experimental setup below $180 \mathrm{~K}$; the change of slope about $330 \mathrm{~K}$ is attributed to the $\mathrm{Fe}^{2+} / \mathrm{Fe}^{3+} \mathrm{CO}^{30}$

These preliminary characterizations, in line with those reported in the literature, confirm the quality of the present sample, which was thus further investigated in details by electron, neutron, and synchrotron x-ray diffraction.

\section{B. Analysis of the neutron (3T2) and x-ray (CRISTAL) diffraction data}

The crystal structure of $\mathrm{LuFe}_{2} \mathrm{O}_{4}$ was first refined in the $R \overline{3} m$ space group ${ }^{7}$ using the neutron diffraction data (Fig. 3), which allow reliable refinement of atomic displacement parameters (ADP) for all species. It shows that the ADP of O(2) and Lu are large $\left(B_{\text {iso }} \cong 2.0\right.$ and $1.1 \AA^{2}$, respectively) but rather isotropic for $\mathrm{O}(2)\left(u_{11} \cong u_{33} \cong 0.035 \AA^{2}\right)$, whereas for lutetium $u_{33}\left(\cong 0.039 \AA^{2}\right)$ it is ten times larger than $u_{11}\left(\cong 0.004 \AA^{2}\right)$; the introduction of anisotropy improves clearly the fit (from 9.7 to 4.8 and from 3.77 to 2.5 for $\chi^{2}$ and $R_{\mathrm{p}}$, respectively). Dealing with this last point, equivalent statistic agreement factors are obtained by splitting the lutetium position from $3 a\left(\begin{array}{lll}0 & 0 & 0\end{array}\right)$ to $6 c(00 z \cong 0.007)$, which resulted in $B_{\text {iso }} \cong 0.19 \AA^{2}$ for this position. This signature of disorder in the compact $\left[\mathrm{LuO}_{2}\right]_{\infty}$ layer has been reported earlier in the $\mathrm{LuFe}_{2} \mathrm{O}_{4}$ single crystal study of Isobe et al.; $;^{7}$ the splitting of the $\left[\mathrm{LuO}_{2}\right]_{\infty}$ layer mode, observed from infrared response at $T_{\mathrm{CO}}$, is attributed to charge-rich and charge-poor proximity effects resulting from an antipolar ordering of the $\left[\mathrm{Fe}_{2} \mathrm{O}_{4}\right]_{\infty}$ layers in Ref. 31 . Horibe et al., in the investigation of $\mathrm{YFe}_{2} \mathrm{O}_{4},{ }^{23}$ claimed that the lattice distortion of the $\left[\mathrm{YO}_{2}\right]_{\infty}$ layer plays a crucial role in the stabilization of long-periodic superstructures. Similarly,
Subramanian et al. ${ }^{18}$ describe a charge-ordered state made of alternating layers of $\mathrm{Fe}^{2+}$ and $\mathrm{Fe}^{3+}$ species, in which incommensurate superstructures may arise from the size mismatch between the layers and/or accommodation of the $\mathrm{Lu}$ sheets. The study of disorder in the $\left[\mathrm{LuO}_{2}\right]_{\infty}$ layer ${ }^{32}$ reveals that the partial $\mathrm{Y}$ or Er for Lu substitution has a strong effect on the distortion of the $\mathrm{FeO}_{5}$ polyhedron that probably influences the ferroelectric properties. Refining the occupancy of atomic sites from NPD data does not improve the modeling and does not evidence any deviation from the ideal $\mathrm{LuFe}_{2} \mathrm{O}_{4}$ composition.

The extra peak (i.e., non-indexed in $R \overline{3} m$ ) that is highlighted by a star in the $300 \mathrm{~K} \mathrm{NPD}$ pattern (Fig. 3) is associated with $\mathrm{CO}$, as confirmed by its disappearance between 300 and $350 \mathrm{~K}$ (inset of Fig. 3). ${ }^{33}$ The charge-order state, not introduced in the diffraction data modeling, is supported also by the Mössbauer and ED results given in the following sections.

Analysis of the synchrotron data with the $R \overline{3} m$ model leads to similar results, with an indication of possible disorder of $\mathrm{Lu}$ cations. Nevertheless a close inspection of the diffractogram suggests a very subtle splitting of several Bragg peaks [inset (i) of Fig. 4], indicative of a lowering of the symmetry. The monoclinic $C 2 / m$ space group, subgroup of $R \overline{3} m^{34}$ through the relation $\overrightarrow{\mathrm{a}}_{M}=\overrightarrow{\mathrm{a}}_{R}-\overrightarrow{\mathrm{b}}_{R}, \overrightarrow{\mathrm{b}}_{M}=\overrightarrow{\mathrm{a}}_{R}+\overrightarrow{\mathrm{b}}_{R}$, and $\overrightarrow{\mathrm{c}}_{M}=$ $\left(\overrightarrow{\mathrm{c}}_{R}-\overrightarrow{\mathrm{a}}_{R}+\overrightarrow{\mathrm{b}}_{R}\right)$ 3, was then used for subsequent Rietveld analysis, leading to $a_{M} \cong 5.9563 \AA, b_{M} \cong 3.4372 \AA$, and $c_{M} \cong 8.6431 \AA$ with $\beta \cong 103.24^{\circ}$ [Fig. 1(b)]. In the following the indices " $M$ " and "R" will be used for the monoclinic and rhombohedral cells, respectively. Lowering the symmetry does not decrease the Debye-Waller factors of the lutetium cations nor their anisotropy, which suggests that the disorder takes place inside the $\left[\mathrm{LuO}_{2}\right]_{\infty}$ layer. Introducing anisotropic strain fails to reproduce perfectly the $(h k l)$-dependent broadening of the Bragg peaks, in particular for the $(00 l)$ peaks that are in addition clearly asymmetric. Unfortunately the monoclinic

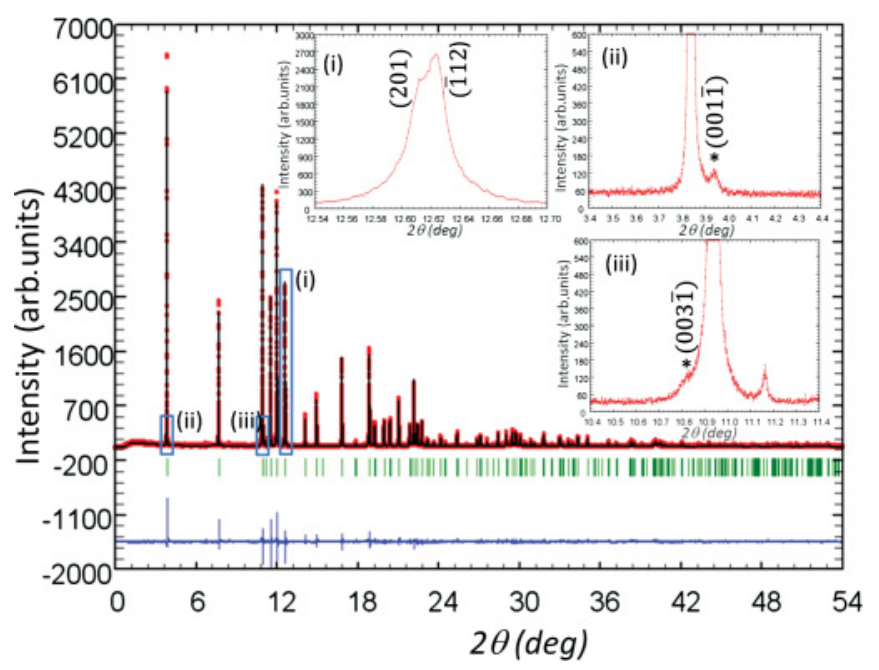

FIG. 4. (Color online) Rietveld refinement of the $300 \mathrm{~K}$ synchrotron x-ray data. Insets: (i) enlargement of the $\left(\begin{array}{lll}0 & 1 & 5\end{array}\right)_{R}$ Bragg reflection evidencing the monoclinic distortion; (ii) and (iii) enlargements showing the $\vec{q} 1$ modulation superstructure peaks indicated by stars. Peak indexations correspond to the monoclinic cell. 
TABLE I. Structural parameters of $\mathrm{LuFe}_{2} \mathrm{O}_{4}$ at $300 \mathrm{~K}$ (from synchrotron diffraction data Rietveld refinement).

\begin{tabular}{lc}
\hline \hline Space group & $C 2 / m\left(\mathrm{n}^{\circ} 12\right)$ \\
\hline Cell parameters & \\
$a(\AA)$ & $5.9563(1)$ \\
$b(\AA)$ & $3.4372(1)$ \\
$c(\AA)$ & $8.6431(1)$ \\
$\beta\left({ }^{\circ}\right)$ & $103.24(1)$ \\
Cell volume $\left(\AA^{3}\right)$ & $172.24(1)$ \\
Atomic positions & \\
Lu $(2 a)$ & $0,0,0$ \\
Fe $(4 i)$ & $0.2084(9), 0,0.6459(1)$ \\
$\mathrm{O}(1)(4 i)$ & $0.125(3), 0,0.3843(5)$ \\
$\mathrm{O}(2)(4 i)$ & \\
Isotropic B (in $\left.\AA^{2}\right)$ & $0.88(1)$ \\
Lu & $0.46(2)$ \\
Fe & \\
Agreement factors & 5.35 \\
$R_{\text {Bragg }}(\%)$ & 1.66 \\
$\chi^{2}$ & \\
\hline \hline
\end{tabular}

distortion cannot be resolved on the NPD data, and no new information can be inferred concerning the oxygen atoms. The atomic positions in $C 2 / m$ refined with CRISTAL data (Table I) are, thus, to be taken with care. The discussion concerning the selected interatomic distances reported in Table II is also difficult; nevertheless, distances extracted from calculations in $C 2 / m$ with synchrotron data and in $R \overline{3} m$ with neutron data are comparable. Iron shows an unusual coordination, surrounded by four $\mathrm{O}$ at about $2.0 \AA$ : one $\mathrm{O}(1)$ lying in between the $\mathrm{Lu}$ and $\mathrm{Fe}$ layers and three $\mathrm{O}(2)$ forming the basal plane of the triangular pyramid around the iron atoms. The fifth oxygen involved in the Fe-coordination polyhedron lies in the other Fe layer at a longer distance $(\cong 2.2 \AA)$. Compared with the rhombohedral structure, the monoclinic distortion introduces anisotropy in the six interatomic $\mathrm{Fe}$ Fe distances in the basal triangular plane, but refinements show that these distances actually stay the same, within the experimental errors $\left(\mathrm{Fe}-\mathrm{Fe}_{\text {(intra) }}\right.$, Table II). In contrast, when considering the buckled honeycomb lattice (also called $\mathrm{W}$-layer in this kind of compound ${ }^{35}$ ), the monoclinic distortion leads to a more pronounced anisotropy of the shorter $\mathrm{Fe}-\mathrm{Fe}$

TABLE II. Selected interatomic distances (in $\AA$ ) and multiplicity.

\begin{tabular}{|c|c|c|}
\hline \multirow[t]{2}{*}{$\mathrm{Lu}-\mathrm{Lu}$} & $3.4371(1)$ & $x 2$ \\
\hline & $3.4384(1)$ & $x 4$ \\
\hline \multirow[t]{2}{*}{$\mathrm{Fe}-\mathrm{Fe}{ }_{\text {(intra) }}$} & $3.4371(1)$ & $x 2$ \\
\hline & $3.438(7)$ & $x 4$ \\
\hline \multirow[t]{2}{*}{$\mathrm{Fe}-\mathrm{Fe}_{\text {(inter) }}$} & $3.107(5)$ & $x 1$ \\
\hline & $3.183(3)$ & $x 2$ \\
\hline \multirow[t]{2}{*}{$\mathrm{Lu}-\mathrm{O}(1)$} & $2.313(11)$ & $x 2$ \\
\hline & $2.172(5)$ & $x 4$ \\
\hline $\mathrm{Fe}-\mathrm{O}(1)$ & $1.995(4)$ & $x 1$ \\
\hline $\mathrm{Fe}-\mathrm{O}(2)$ & $2.201(4)$ & $x 1$ \\
\hline $\mathrm{Fe}-\mathrm{O}(2)_{\text {in plane }}$ & $2.028(13)$ & $x 2$ \\
\hline $\mathrm{Fe}-\mathrm{O}(2)_{\text {in plane }}$ & $1.95(2)$ & $x 1$ \\
\hline
\end{tabular}

distances between triangular planes $\left(\mathrm{Fe}-\mathrm{Fe}_{(\text {inter }}\right.$, Table II). This symmetry lowering is in agreement with the transition from $R \overline{3} m$ to $C 2 / m$ observed at $320 \mathrm{~K}$ by Vitucci $e t$ al. in their infrared study. ${ }^{37}$ Note here that a description of $\mathrm{LuFe}_{2} \mathrm{O}_{4}$ by means of the $C 2 / \mathrm{m}$ space group has been previously used by several groups ${ }^{31,33,36}$ but in connection with an enlarged cell accounting for $\mathrm{CO}$.

An enlargement of the low-angle part of the x-ray pattern shows very small extra peaks [insets (ii) and (iii) of Fig. 4], that can be indexed with the $\vec{q}_{1}=\left[\begin{array}{llll}0.532 & 0 & 0.124\end{array}\right]$ modulation vector determined from ED (see in the following), which does not correspond to that expected for $\mathrm{CO}$, based on previous reports. ${ }^{15-18}$ In order to account for these extra peaks in the calculations, modulations of atomic parameters were introduced using the $C 2 / m(\alpha 0 \gamma) 00$ super-space group. Clearly, modulating the occupancies of $\mathrm{Fe}, \mathrm{Lu}$, and $\mathrm{O}$ fails to reproduce both peaks simultaneously, while the modulation of atomic positions, along the direction perpendicular to the $(a, b)$ plane, transfers intensity to these peaks. Three types of modulation waves can be used to model the positional disorder: single sine harmonic, sawtooth, or zigzag. The single sine harmonic does not reproduce the peak intensities properly; because only two weak peaks are available, it was not possible to discriminate between the two remaining possibilities, and the sawtooth type modulation wave was arbitrarily chosen. For the same reason, the study was realized, in a first step, by checking models without refining modulation parameters until correct intensities are obtained. The amplitude of the modulation is unrealistic for $\mathrm{O}$ atoms (equivalent displacement $\delta z \cong 0.2)$, and it is also the case for $\mathrm{Fe}$ but with a much smaller value $(\delta z \cong 0.05)$; the best model corresponds to the modulation of the position of Lu. The modulation parameters of Lu were therefore refined along $a_{M}$ and $c_{M}$ simultaneously, and the fitted values of $\delta x \cong 0.007$ and $\delta z \cong 0.02$ correspond to the displacement obtained with a split model. Refining the three components of the modulation wave $(\delta x, \delta y, \delta z)$ of $\mathrm{Lu}$ does not improve the refinement.

To summarize this section, the $\mathrm{LuFe}_{2} \mathrm{O}_{4}$ sample under study is a single phase, the composition is very close to the expected one (no deviation of the ideal composition was evidenced by neutron and x-ray diffraction data), a small monoclinic distortion is observed, and some details are indicative of displacive modulations, particularly on the Lu site. Even if the quality of the fits is rather good, as illustrated by the agreement factors and Figs. 3 and 4, some points remain unclear and other techniques of characterization are needed to push further the analysis.

\section{Mössbauer spectroscopy}

The crucial point evidenced with Mössbauer spectroscopy is the coexistence of $\mathrm{Fe}^{2+}$ and $\mathrm{Fe}^{3+}$ species at $\mathrm{RT}$ with the expected ratio $\mathrm{Fe}^{2+} / \mathrm{Fe}^{3+}=1$ (within a $2 \%$ accuracy), also confirming the stoichiometric oxygen content. The Mössbauer spectrum obtained at $300 \mathrm{~K}$ (Fig. 5) was fitted with a BlumeTjon model ${ }^{38}$ for $\mathrm{Fe}^{2+}$ and $\mathrm{Fe}^{3+}$ relaxation, similar to the one used by Tanaka et al. ${ }^{39}$ The hyperfine parameters, which are reported in Table III, are in perfect agreement with those reported by $\mathrm{Xu}$ et al. for $\mathrm{LuFe}_{2} \mathrm{O}_{4}$ crushed single crystals. ${ }^{36}$ Additionally, the use of this relaxation model shows that the 


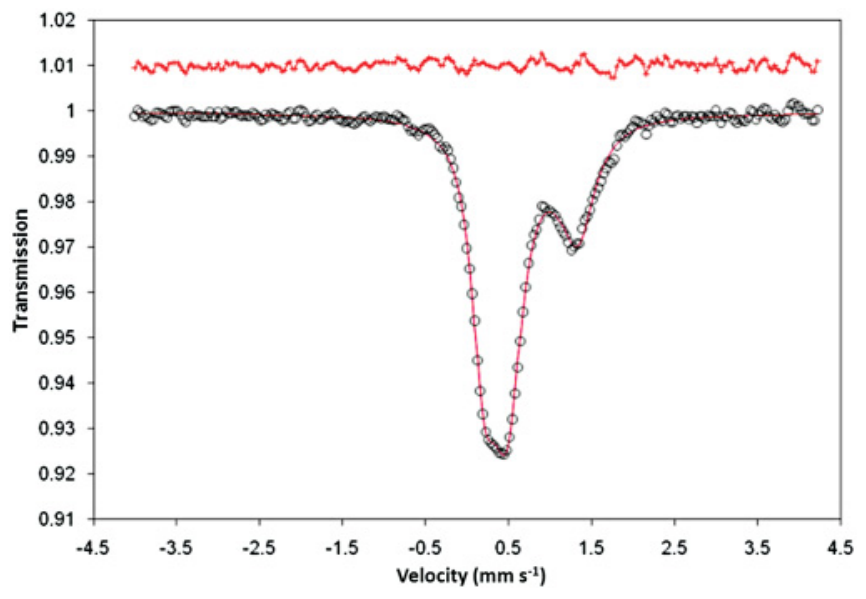

FIG. 5. (Color online) $300 \mathrm{~K}$ Mössbauer spectrum fitted using a relaxation model for the $\mathrm{Fe}^{2+}$ and $\mathrm{Fe}^{3+}$ contributions.

$\mathrm{Fe}^{2+} / \mathrm{Fe}^{3+}$ hopping frequency is found to be $1.4 \mathrm{MHz}$, which invalidates the presence of $33 \%$ of $\mathrm{Fe}^{2.5+}$ (characterized by a hopping frequency of $1 \mathrm{GHz}$ ) below $T_{\mathrm{CO}}$ suggested in Ref. 15. This experiment confirms that the compound does not show magnetic order at RT. However, because of the unusual bipyramidal " $\mathrm{O}_{4+1}$ " environment of iron, it is difficult to push the analysis further to determine a difference between the coordination of $\mathrm{Fe}^{2+}$ and $\mathrm{Fe}^{3+}$. The present observations are in agreement with the resonant $\mathrm{x}$-ray diffraction at the $\mathrm{Fe}-\mathrm{K}$ edge showing a full charge separation into $\mathrm{Fe}^{2+}$ and $\mathrm{Fe}^{3+} .40$

\section{ED}

The TEM study confirmed the good crystalline quality of the grains and revealed two important new features: the existence of a new type of ordering besides the ones previously reported by numerous authors, ${ }^{15,27,41-43}$ and original macroand nanostructural states that will be detailed elsewhere. ${ }^{44}$ In fact, due to the complexity of the TEM data, only results shedding light on the behavior described previously are given, particularly those dealing with the new modulation.

\section{RT study}

The reconstruction of the reciprocal space of about 70 crystallites was carried out by tilting around the crystallographic axes of the $R \overline{3} m$ and $C 2 / m$ cells. It evidenced the coexistence of several systems of reflections: intense spots associated with the subcell and less intense ones related to the presence of modulated structures.

The system of intense reflections appears very close to what is expected for the $R \overline{3} m$ structure, with the conditions of reflection and the hexagonal-like arrangement of the spots in the $[001]_{R}$ ED pattern. However, taking into account the synchrotron data, particular attention was paid to details pointing toward a monoclinic distortion. For a large number of crystallites, the distortion is hardly detectable, but the presence of satellites clearly implies a violation of the rhombohedral symmetry. As a consequence, the monoclinic $C 2 / \mathrm{m}$ cell described previously will be used for the explanation of the TEM results. To go further, the contrast of the HRTEM images, recorded in areas associated with satellite-free ED patterns, was interpreted with the help of the simulated focal series calculated for the rhombohedral and monoclinic cells. Both image series are very similar; slight differences can only be detected at the level of the Lu layers for a limited number of focus values. A typical image is given in Fig. 6(a) and compared to the calculated image (focus value $-600 \AA$ and crystal thickness $34 \AA ; C 2 / m$ space group). The positions of the iron atoms of the bi-layers appear as brighter dots, whereas the lutetium positions correspond to the grey spots in between the brighter dots of the zigzagging row.

The lattice images in a modulated zone reveal the existence of stacking defects, along the direction perpendicular to the layer plane [Fig. 6(b)]. These planar defects induce weak diffuse streaks, sometimes observed along $\vec{c}_{M}^{*}$ in the diffraction patterns. It is important to note that these defective slices have a local thickness $c_{\text {def }}=m \times 1 / 3 c_{R}$ and are not caused by the insertion of single iron layer, according to the growth mechanism reported for the $\mathrm{RFe}_{2} \mathrm{O}_{4}\left(\mathrm{RFeO}_{3}\right)_{\mathrm{m}}$ family $(m=$ $0,1 \ldots) .{ }^{47-49}$ The TEM investigation, as discussed in Ref. 44, shows that they often affect two adjacent cells, involving a local periodicity $c_{\text {def }} \approx 16.8 \AA(m=2)$, as illustrated by the defects indicated by white arrows in Fig. 6(b). The two main origins of the planar defects are nano-twinning, which often run over one or two cells only and gliding between two $\mathrm{Fe}$ bi-layers and the lutetium layers.

The extra spots observed in the ED patterns of most crystallites are associated with modulated structures characterized by the vectors $\vec{S}=h \vec{a}^{*}+k \vec{b}^{*}+l \vec{c}^{*}+m \vec{q}$. Three types of modulated structures, indicated by the vectors $\vec{q}_{1}, \vec{q}_{2}$, and $\vec{q}_{3}$, occur in the form of a unique modulation in certain crystals or as coexisting modulations in other ones. This point is illustrated viewing the crystallites exhibiting all three modulations along zone axes perpendicular and parallel to the layer planes.

(a) [hk0] ED patterns. Five [hk0] ED patterns recorded through a $90^{\circ}$ tilt around the $\vec{c}_{M}^{*}$ axis are given from $[100]_{M}$ [Fig. 7(a)] to [010] $]_{M}[$ Fig. 7(e)].

Modulation I $\left(\vec{q}_{1}\right)$. This system of satellites is the most frequently observed in our sample. It is described through a modulation vector $\vec{q}_{1}=\alpha_{1} \overrightarrow{\mathrm{a}}_{M}^{*}+\gamma_{1} \vec{c}_{M}^{*}$, which, to our

TABLE III. Hyperfine parameters for the $\mathrm{Fe}^{2+}, \mathrm{Fe}^{3+}$ components fitted from the Mössbauer spectrum in Fig. 5. Absolute errors are given in brackets and refer to the last digit.

\begin{tabular}{lccccc}
\hline \hline & $\begin{array}{c}\text { Line width } \\
(\mathrm{mm} / \mathrm{s})\end{array}$ & $\begin{array}{c}\delta \text { Isomer shift } \\
(\mathrm{mm} / \mathrm{s})\end{array}$ & $\begin{array}{c}\Delta \text { Quadrupole } \\
\text { Splitting (mm/s) }\end{array}$ & $\begin{array}{c}\mathrm{Hopping} \\
\text { freq. (MHz) }\end{array}$ & $\begin{array}{c}\mathrm{Fe}^{3+} /\left(\mathrm{Fe}^{2+}+\mathrm{Fe}^{3+}\right) \\
\text { phase fraction }\end{array}$ \\
\hline $\mathrm{Fe}^{3+}$ & $0.26(3)$ & $0.33(1)$ & $0.33(2)$ & $1.4(5)$ & $0.51(2)$ \\
$\mathrm{Fe}^{2+}$ & $0.40(5)$ & $0.90(2)$ & $0.90(2)$ & & \\
\hline \hline
\end{tabular}


(6a)

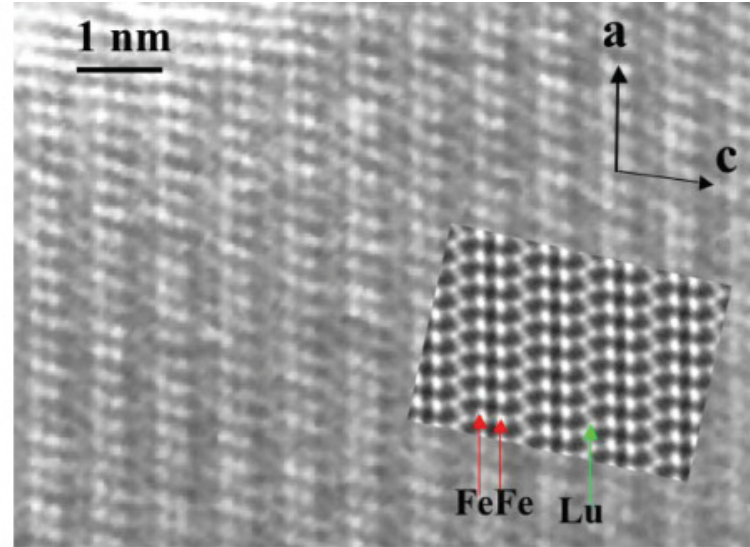

(6b)

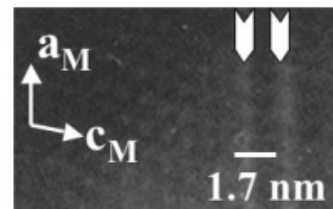

(6c)

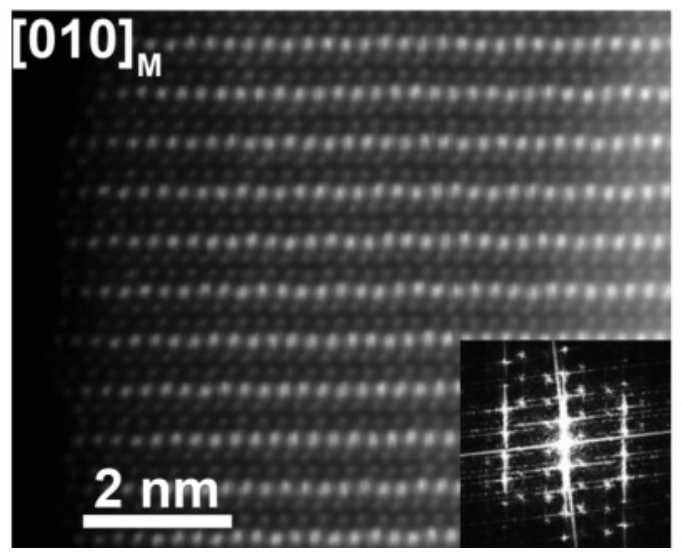

FIG. 6. (Color online) $[010]_{M}$ images. (a) HREM experimental and calculated images of a nonmodulated zone. (b) Lattice image of a modulated zone showing a large area where the simple commensurate approximant is established. The defects are indicated by white marks. (c) $[010]_{M}$ HAADF-STEM image of a modulation I zone; the corresponding Fourier transform is shown in the inset.

knowledge, has never been reported to date. The $[010]_{M}$ ED pattern, enlarged in Fig. 8(a), is indexed using $\mathrm{hklm}$ indices $(m \neq 0$ indicate satellites). In this example the satellites are nearly aligned along the $[401]_{M}^{*}$ and $[20 \overline{3}]_{M}^{*}$ directions of the monoclinic reciprocal subcell and are also aligned parallel to the $[60 \overline{2}]_{M}^{*}$ direction (as the $\overline{2} 0 \overline{1} 1$ and $40 \overline{1} \overline{1}$ satellites highlighted by circles) at positions $1 / 3,2 / 3$, and 1 along $\vec{c}_{M}^{*}$. Such arrangement of the satellites with regard to the basic reflections evocates a commensurate modulation with $\alpha_{1}=$ $4 / 7$ and $\gamma_{1}=1 / 7$, but tiny deviations from commensurability are observed with the average measured values $\alpha_{1}=0.55 \pm$ 0.02 and $\gamma_{1}=0.13 \pm 0.01$. The very small and broad extra peak

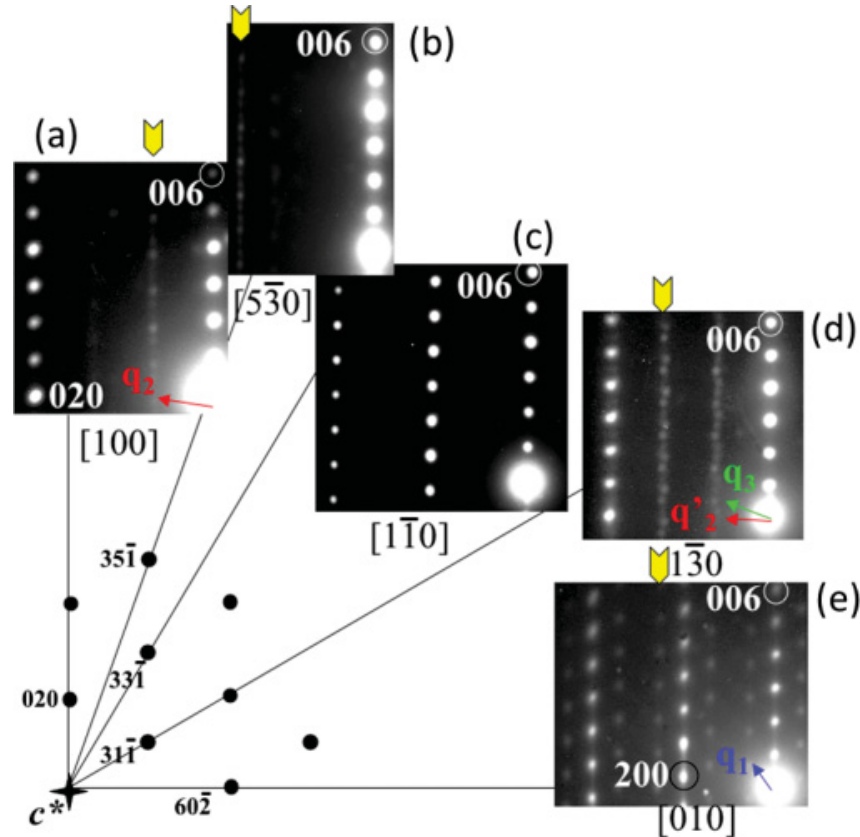

FIG. 7. (Color online) Three types of modulation observed through the reconstruction of the reciprocal subcell by tilting around $\vec{c}_{M}^{*}$. The lines represent the relative tilting angles (from $0^{\circ}$ for the $[010]_{M}$ pattern up to $90^{\circ}$ for the $[001]_{M}$ ), and the yellow arrows the lines of satellites running along $\vec{c}_{M}^{*}$. The weak satellites on the $[1 \overline{1} 0]_{M}$ pattern (c) are ascribed to a domain with slightly different $\vec{q}_{1}$ captured by a selected area aperture.

observed in the RT synchrotron data can be associated with this modulation, with a refined value of [0.53 00.124$]$ for $\vec{q}_{1}$.

The $[010]_{M}$ and $[1 \overline{1} 0]_{M}$ ED patterns are not equivalent, as they should be in $R \overline{3} \mathrm{~m}$ : the satellites are clearly visible on the first pattern at $0^{\circ}\left([010]_{M}\right.$ in Fig. $\left.7(\mathrm{e})\right)$. On the $[1 \overline{1} 0]_{M}$ in Fig. 7(c) (equivalent to the $[010]_{R}$ pattern), the satellites are hardly visible and have slightly different positions. It shows undoubtedly that these reciprocal lattice sections are not equivalent and confirms the loss of rhombohedral symmetry.

The $[010]_{M}$ lattice images [Fig. 6(b)] evidence intense contrast variations associated with this modulation I; they are established over rather large areas of the order of several hundred nanometers. The $[010]_{M}$ HAADF-STEM image in Fig. 6(c) provides more details on the atomic nature of this modulation. The brighter dots on the image are the projections of the Lu columns, and the less bright dots are the projections of the Fe columns according to their $Z$ values. The Fourier transform shows the corresponding satellite reflections. The $\mathrm{Lu}$ layers clearly show a wiggling shape that suggests that the Lu atomic displacements have a significant contribution to the modulation, in agreement with the results of the Rietveld refinements.

Modulation II $\left(\vec{q}_{2}\right)$ and modulation III $\left(\vec{q}_{3}\right)$. The second type of modulation (II) is observed in the $[100]_{M}$ ED pattern [Fig. 7(a)] where all the satellites are indexed using a vector $\vec{q}_{2}=\beta_{2} \vec{b}_{M}^{*}+\gamma_{2} \vec{c}_{M}^{*}$, with $\beta_{2}=2 / 3$ and $\gamma_{2}=1 / 6$ (and $\gamma_{2}=5 / 6$ owing to the existence of twin planes perpendicular to $\vec{c}_{M}^{*}$ ). The modulated structure II is commensurate, but here 
(a)

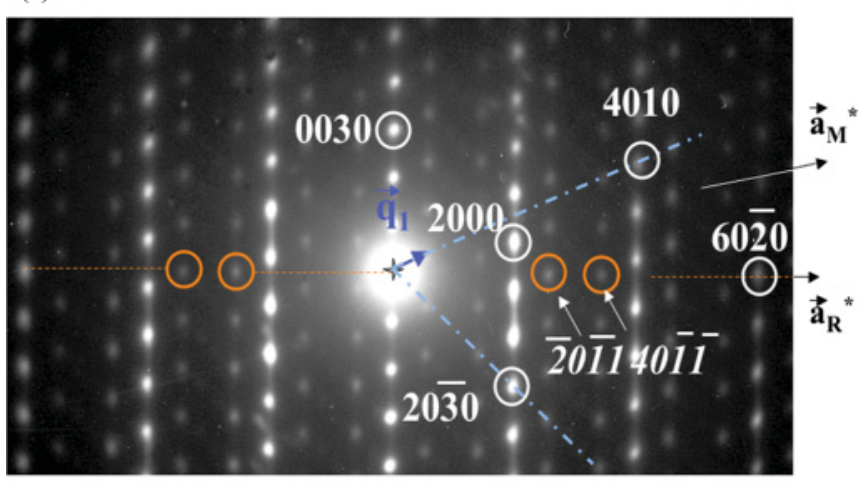

(b)

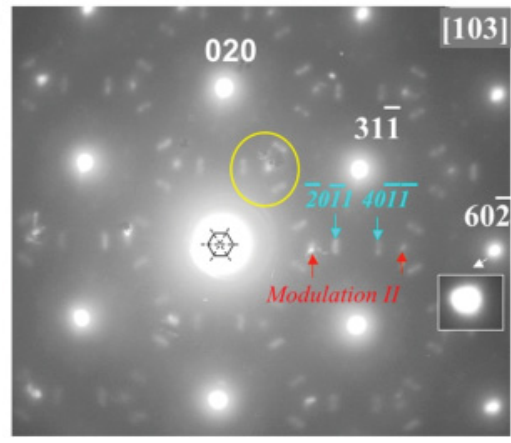

FIG. 8. (Color online) ED patterns with indexation using four indices; the numbers in italics are associated with the satellites: blue and red for modulations I and II, respectively. (a) $[010]_{M}$ ED pattern. (b) Twinning phenomena in the $[103]_{M}$ ED pattern (equivalent to the $[001]_{R}$ ). The reflection $60 \overline{2}$ is enlarged in the yellow rectangle.

again deviations from $\beta_{2}=2 / 3$ are sometimes observed by tilting over $60^{\circ}$, as in the $[1 \overline{3} 0]_{M}$ pattern [Fig. 7(d)] [equivalent to Fig. 7(a) in a $R \overline{3} m$ cell]. The positions of the satellites are slightly displaced, involving an incommensurate modulation with $\vec{q}_{2}^{\prime}=\beta_{2} \vec{b}_{M}^{*}+\gamma_{2} \vec{c}_{M}^{*}$ and $\beta_{2}=2 / 3-\varepsilon$ and $\gamma_{2}=1 / 6$. Twinning phenomena $\left(\gamma_{2}=1 / 6\right.$ and 5/6) lead to zig-zagging rows of satellites and diffuse streaks along $\vec{c}_{M}^{*}$. Another more intense set of satellites coexists with $\vec{q}^{\prime}{ }_{2}$ in this $[1 \overline{3} 0]_{M}$ pattern, associated with the commensurate vector of a third modulation (III) $\vec{q}_{3}=\beta_{3} \vec{b}_{M}^{*}+\gamma_{3} \vec{c}_{M}^{*}$ with $\beta_{2}=2 / 3$ and $\gamma_{3}=1 / 2$. The coexistence of $\vec{q}^{\prime}{ }_{2}$ and $\vec{q}_{3}$ reinforces the zig-zagging effect observed in Fig. 7(d). The presence of three modulations (vectors $\vec{q}_{2}, \vec{q}_{2}^{\prime}$, and $\vec{q}_{3}$ ) in one crystallite can be correlated to the small dimensions of their existence domains. Tilting over an angle of $90^{\circ}$ induces indeed the overlap of the different zones.

The $[100]_{M}$ and equivalent lattice images (not presented here) of modulated structures II or III exhibit a striped contrast characteristic of crossing twin domains, of the order of a few nanometers, without coherent boundaries.

The components along $\vec{c}_{M}^{*}$ of the propagation vectors $\gamma_{2} \vec{c}_{M}^{*}$ with $\gamma_{2}=1 / 6$ and $\gamma_{3} \vec{c}_{M}^{*}$ with $\gamma_{3}=1 / 2$ are equivalent to $1 / 2 \vec{c}_{R}^{*}$ and $3 / 2 \vec{c}_{R}^{*}$, respectively. These modulations can therefore be directly compared to those previously reported in the $R \overline{3} m$ cell for the modulations II $^{23,45}$ and III. ${ }^{11,33,43,46}$

(b) $[103]_{M}$ ED pattern. On the $[103]_{M}$ ED patterns (equivalent to $[001]_{R}$ ) of thin crystals having incommensurate modulation vectors, the only spots are the $h 0 l 0$ reflections, which adopt the "hexagonal-like" arrangement expected for the $\mathrm{C} 2 / \mathrm{m}$ monoclinic subcell. As the modulation is nearly commensurate and/or with diffuse streaks along $\vec{c}_{M}^{*}$, weak additional spots are observed, as in the complex $[103]_{M}$ ED pattern given in Fig. 8(b), which illustrates the coexistence of the modulated systems (vectors $\vec{q}_{1}, \vec{q}_{2}, \vec{q}_{2}^{\prime}$, and $\vec{q}_{3}$ ) and twinning phenomena. The distortion with regard to a perfect hexagonal arrangement of the basic $h 0 l 0$ spots results from tiny variations of the interplanar distances $d \overline{6} 02$ (the enlargement in the insert shows that the reflection $60 \overline{2}$ is not a single node), $d 33 \overline{1}$ and $d \overline{3} 31$, whereas the angles between the three corresponding vectors remain very close to $60^{\circ}$. Along these three directions additional reflections are observed, forming a hexagonal crown around the basic spots as a result of the twinning phenomena associated with the symmetry lowering. The satellites indicated by blue arrows belong to modulation I (indexed $\overline{2} 0 \overline{1} 1$ and $40 \overline{1} \overline{1}$ ). Note that, in this example, they are elongated perpendicularly to the direction [ $[\overline{6} 02]^{*}$, suggesting that the modulation vector could exhibit a component along $\vec{b}_{M}^{*}$. Two nodes appear in the elongated spots, leading to $\vec{q}^{\prime}{ }_{1}=$ $\alpha_{1} \vec{a}_{M}^{*}+\beta_{1} \vec{b}_{M}^{*}+\gamma_{1} \vec{c}_{M}^{*}$ and $\alpha_{1} \approx 4 / 7, \beta_{1} \approx 0.05$, and $\gamma_{1} \approx 1 / 7$. However, such a phenomenon has been observed in a minority of crystals, and this small translation of the satellites along $\vec{b}_{M}^{*}$ explains why, in the [010] $]_{M}$ ED patterns, the satellites are sometimes visible or observed with higher intensity by a small tilting of the crystallite.

As mentioned previously, the $\vec{q}_{2}$ (and $\vec{q}_{2}{ }^{\prime}$ ) and $\vec{q}_{3}$ modulations have been previously reported in TEM studies ${ }^{11,23-46}$ in the rhombohedral cell: our observations evidence similar CO mechanisms and furthermore support a loss of the $R \overline{3} \mathrm{~m}$ symmetry. Deviations from the commensurate value $\beta_{2}=2 / 3$ have previously been reported by $\mathrm{ED}^{15}$ and $\mathrm{x}$-ray and neutron diffraction ${ }^{16}$ and in the $\mathrm{Yb}$-based system. ${ }^{50}$ They are also reported as a function of temperature, ${ }^{15,27}$ electric and magnetic fields, ${ }^{41,42}$ or oxygen content in the Y-compounds. ${ }^{21,51}$ The $\vec{q}_{2}$ (and $\vec{q}_{2}{ }^{\prime}$ ) and $\vec{q}_{3}$ modulations, commonly attributed to $\mathrm{CO}$ in $\mathrm{LuFe}_{2} \mathrm{O}_{4}$, are compatible with the extra peak that we observe around $29.22^{\circ}$ in $2 \theta(d \approx 2.43 \AA)$ on the $3 \mathrm{~T} 2$ data at RT, which disappears in the $350 \mathrm{~K}$ measurement (inset of Fig. 4). This motivated the temperature study that follows, in which the effect of temperature upon the ED patterns was investigated, with a special focus on the new modulation I.

\section{ED vs temperature}

The $[010]_{M}$ and $[100]_{M}$ zone axes, exhibiting the modulations I and II, respectively, have been selected for the ED study vs temperature. The crystals were heated up to $343 \mathrm{~K}$ (i.e., $>T_{\mathrm{CO}}$ ), cooled down to RT and heated again, progressively, up to $773 \mathrm{~K}$ and last, cooled down to RT.

$[100]_{M}$ zone: modulation $\vec{q}_{2}$ (Fig. 9, RT). When the crystal is heated in situ up to $343 \mathrm{~K}$ (Fig. 9, $343 \mathrm{~K}$ ), the satellites and diffuse intensity lines at $02 / 31 / 6$ completely vanish, in agreement with the disappearance of $\mathrm{CO}$, and cooling back to RT restores the diffuse intensity [Fig. 9, RT (bottom)].

$[010]_{M}$ zone: modulation $\vec{q}_{1}$ (Fig. 9). Starting from a RT modulation vector $\vec{q}_{1} \approx 0.53 \vec{a}_{M}^{*}+0.12 \vec{c}_{M}^{*}$ with very weak diffuse lines running along $[20 \overline{3}]_{M}^{*}$ at RT [Fig. 9, RT (top)] and heating up in situ, the satellite reflections do not disappear, but their positions progressively change (Fig. 9, $343 \mathrm{~K}$ and enlargement in the insert). For instance, at $343 \mathrm{~K}$ (temperature of disappearance of the modulations II and III), 


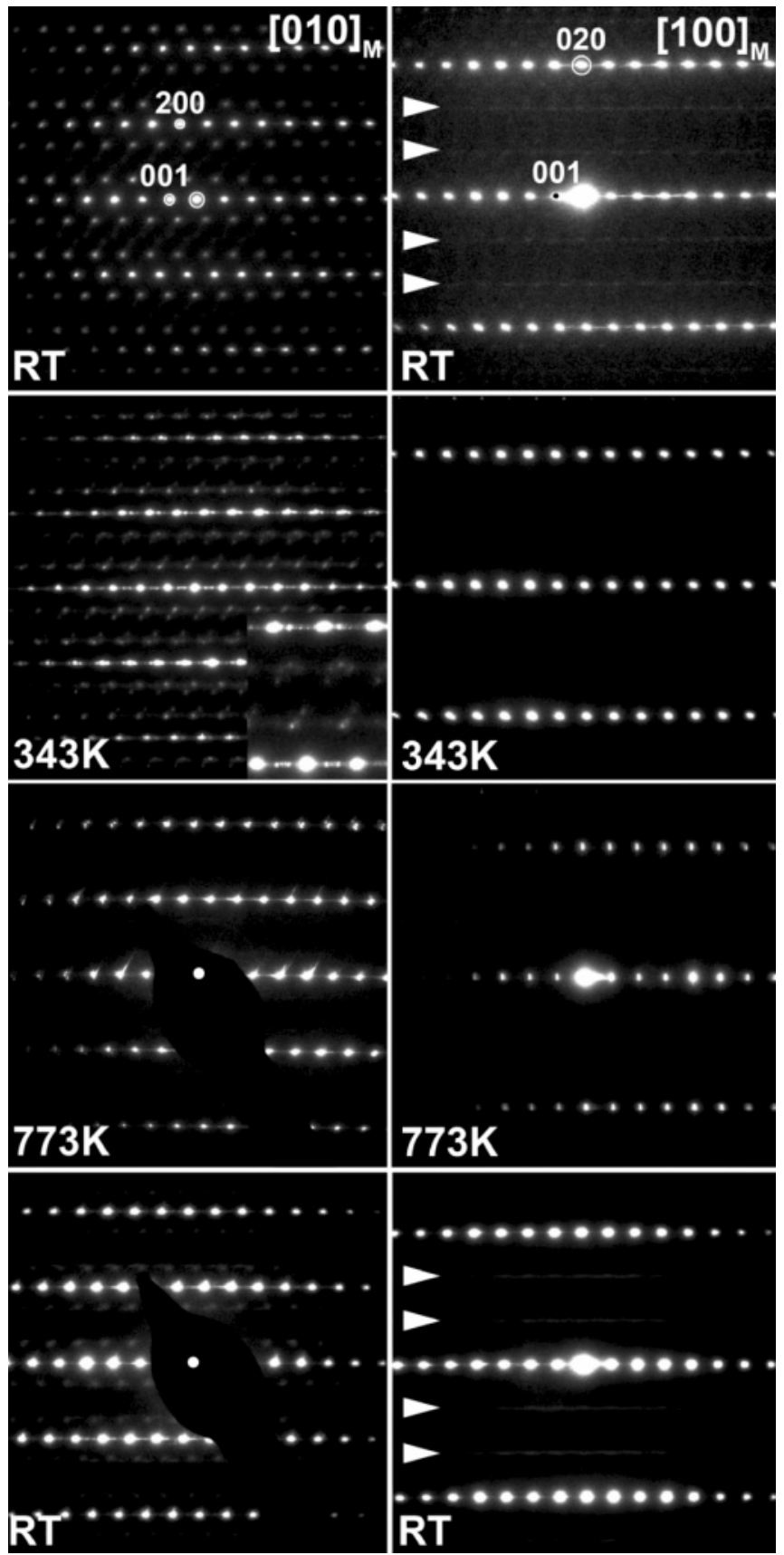

FIG. 9. $[010]_{M}$ and $[100]_{M}$ ED patterns of $\mathrm{LuFe}_{2} \mathrm{O}_{4}$ at (from top to bottom) RT, $343 \mathrm{~K}, 773 \mathrm{~K}$, and after cooling to RT. Diffuse intensity lines due to $\mathrm{CO}$ are marked with arrowheads.

two sets of satellites are now observed, corresponding to $\cong 0.57 \vec{a}_{M}^{*}+0.12 \vec{c}_{M}^{*}$ and $\cong 0.75 \vec{a}_{M}^{*}-0.10 \vec{c}_{M}^{*}$. Moreover, as shown in the enlargement, the diffuse lines along $[20 \overline{3}]_{M}^{*}$ become more intense, signature of an increasing disorder along this direction. Concomitantly, diffuse intensity lines with modulated intensity are also visible along $\vec{c}_{M}^{*}$ that can be caused by closely positioned defective layers associated with these variations in the modulation vectors. Heating up to $773 \mathrm{~K}$ removes all superstructure reflections and leaves the reflections of the basic structure only (Fig. 9, $773 \mathrm{~K}$ ). Cooling down to RT leads to the reappearance of a single system of satellites close to that observed at the beginning of the experiment, without diffuse lines [Fig. 9, RT (bottom)].

\section{DISCUSSION AND CONCLUSION}

The ED study shows that the several systems of satellites are closely connected to a symmetry lowering from rhombohedral to monoclinic. The modulation I, stabilized over larger domains than those corresponding to the modulations II and III, is supposed to act also on the cell symmetry at a temperature above $T_{\mathrm{CO}}$. The intense variations of contrast visible in the $[010]_{M}$ lattice images [Fig. 6(b)] and the displacement of the cationic columns visible in the HAADFSTEM images [Fig. 6(c)] suggest that the ordering phenomena associated with the modulation I involve rather large Lu atomic displacements in the structure.

Modulations II and III are associated with CO: the corresponding satellites disappear at $\cong 330 \mathrm{~K}$, which is the $T_{\mathrm{CO}}$ determined from the resistivity curve, and which is in line with the majority of the previously reported papers on $\mathrm{LuFe}_{2} \mathrm{O}_{4} \cdot{ }^{15,41-43}$ The possible existence of persisting 2D CO up to $530 \mathrm{~K}$, mentioned in Ref. 27, cannot be evidenced in our sample because complex phenomena linked to modulation I are observed vs temperature and will be discussed separately. ${ }^{44}$

Accordingly, the present study shows that the phenomenon which generates the modulation $\vec{q}_{1}$ is reversible and that the mechanism seems to appear/disappear progressively upon decreasing/increasing the temperature. Moreover the state of the sample at a given temperature slightly varies with the history of the sample, first through the components of the modulation vectors $\left(\alpha_{1}\right.$ and $\left.\gamma_{1}\right)$ and second by the diffuse lines which appear along $[20 \overline{3}]_{M}^{*}$ and $\vec{c}_{M}^{*}$.

The TEM observations explain the difficulties encountered in the refinement of the crystal structure using the $\mathrm{x}$-ray and neutron diffraction data, but a coherent picture is obtained by combining these techniques. At RT, the sample exhibits $\mathrm{CO}$ of the $\mathrm{Fe}^{2+}$ and $\mathrm{Fe}^{3+}$ species, in agreement with the Mössbauer study; neutron and x-ray diffraction show no anomaly at the iron position, and disorder only appears on the oxygen positions within the iron layers. This is consistent with a short scale displacement of the oxygen atoms to adapt the $\mathrm{Fe}-\mathrm{O}$ distances vs the oxidation state of iron and could explain the anomalous decrease of the Debye-Waller factor of $\mathrm{O}(2)$ observed after refinement of neutron data between $300 \mathrm{~K}$ and $350 \mathrm{~K}$. In contrast, all other atoms show the expected increase of the Debye-Waller factor with increasing temperature. A charge-ordered superstructure is also consistent with the extra spots in the $[100]_{M}$ ED patterns, corresponding to $\vec{q}_{2}$ and $\vec{q}_{3}$, and the extra peak on the RT neutron data that disappears upon heating to $350 \mathrm{~K}$. This small and broad peak is indeed indexed $1 \overline{1} \overline{1} 1$ and $\overline{1} \overline{1} 31$ using the $[1 / 31 / 31 / 2]$ propagation vector in $R \overline{3} m$, equivalent to $\vec{q}_{2}$ in $C 2 / m$.

The most important result is the observation of a new modulation $\vec{q}_{1}$, which could be correlated with a very small and locally ordered oxygen excess in the structure at RT, referring to the disappearance of this superstructure by annealing. This hypothesis was checked by annealing the sample at $670 \mathrm{~K}$ in vacuum $\left(5.10^{-5}\right.$ bars $)$ for $6 \mathrm{hrs}$. In the ED patterns of the $[010]_{M}$ and equivalent zones recorded by tilting around $\vec{c}_{M}^{*}$, no trace of the modulation I was detected anymore in the 
annealed sample. Moreover, the $[100]_{M}$ and equivalent zones still present the diffuse lines and weak spots of the modulations II and III, which is consistent with the preserved $\mathrm{Fe}^{2+} / \mathrm{Fe}^{3+}$ ratio close to 1 . Indeed, the Mössbauer spectrum at $300 \mathrm{~K}$ of the annealed sample does not evidence a departure from the $\mathrm{Fe}^{2+} / \mathrm{Fe}^{3+}$ ratio $(=1 \pm 0.02)$. The quality of the annealed sample was also checked by x-ray diffraction, and no change was observed in the pattern compared with the as-synthesized one; synchrotron x-ray experiments are planned in the near future to confirm this result and to confirm the disappearance of the small extra peaks characteristic of $q_{1}$. Magnetic properties are not modified by the thermal treatment: the magnetization curve is rather similar to the initial one with the same $T_{\mathrm{N}}$, and only a small decrease of the magnitude of the FC curve is observed.

Similarly, the study of another $\mathrm{LuFe}_{2} \mathrm{O}_{4}$ sample, prepared in similar conditions, lead to very close results to those presented here, including atomic position parameters, ADP factors, and occupancies. The only noticeable difference was observed with regard to the cell parameters, which were slightly larger in the second sample $[a=3.4425(1) \AA, c=25.2601(3) \AA, V=$ 259.25(4) $\left.\AA^{3}\right]$ compared to the first one $[a=3.4412(1) \AA$, $\left.c=25.2412(3) \AA, V=258.85(4) \AA^{3}\right]$, a likely indication of a smaller oxygen content. ${ }^{52,53}$ Moreover, this evolution was previously observed in $\mathrm{LuFe}_{2} \mathrm{O}_{4+\delta} 54$ with the report of the lattice dependence on oxygen stoichiometry in the range $-0.065 \leqslant \delta \leqslant+0.015$. Electron and $\mathrm{x}$-ray synchrotron diffraction of the second sample at RT showed the existence of the monoclinic distortion but the lack of the $\vec{q}_{1}$ modulation. All our results converge therefore toward this hypothesis that the ordering mechanism associated with modulation I involves a tiny oxygen deviation from $\mathrm{O}_{4}$, which is at the limit of detection of our techniques. Further experiments are in progress to control precisely the oxygen stoichiometry of this ferrite. The first results of thermogravimetric analysis show that it is possible to incorporate oxygen in the structure in agreement with pioneer works about lanthanoide ferrites, ${ }^{55,56}$ reporting that for a large amount of oxygen (leading to the formula $\mathrm{LuFe}_{2} \mathrm{O}_{4.5}$ ) a new structure is obtained. Moreover, a possible oxygen nonstoichiometry in $\mathrm{LuFe}_{2} \mathrm{O}_{4}$ was previously proposed in Ref. 57, as linked to the disorder observed in the $\left[\mathrm{LuO}_{2}\right]_{\infty}$ layer, referring to the publications of Nespolo et $a .^{58-60}$ By a charge-distribution analysis of substituted samples belonging to this system, a $\mathrm{Lu}^{3+}-\mathrm{Fe}^{3+}$ repulsion is suggested and related to the particular asymmetry of the $\mathrm{FeO}_{5}$ polyhedron as a possible cause of deviation from the average structure, along with oxygen nonstoichiometry. Iron oxides, especially those containing $\mathrm{Fe}^{2+}$, like in $\mathrm{FeO}, \mathrm{Fe}_{3} \mathrm{O}_{4}$, or some hexaferrites or oxoperovskites, ${ }^{54,61}$ are indeed well known for their nonstoichiometry ability.

\section{ACKNOWLEDGMENTS}

This work was supported by funding from the European Research Council under the 7th Framework Program (FP7), ERC grant $N^{\circ} 246791$ - COUNTATOMS from the Hercules Foundation of the Flemish Government and from the French Agence Nationale de la Recherche (ANR-08-BLAN-0005-01 and JC08-331297). *julie.bourgeois@ensicaen.fr

${ }^{1}$ S. W. Cheong and M. Mostovoy, Nat. Mater. 6, 13 (2007).

${ }^{2}$ D. V. Efremov, J. V. D. Brink, and D. I. Khomskii, Nat. Mater. 3, 853 (2004).

${ }^{3}$ W. Eerenstein, N. D. Mathur, and J. F. Scott, Nature 442, 759 (2006).

${ }^{4}$ J. van den Brink and D. I. Khomskii, J. Phys. Condens. Matter 20, 434217 (2008).

${ }^{5}$ S. Ishihara, J. Phys. Soc. Jpn. 79, 011010 (2010).

${ }^{6}$ N. Kimizuka, E. Muromachi, and K. Siratori, in Handbook on the Physics and Chemistry of Rare Earths, edited by K. A. Gschneider Jr and L. Eyring (Elsevier Science, Amsterdam, 1990), Vol. 13, pp. 283-384.

${ }^{7}$ M. Isobe, N. Kimizuka, J. Iida, and S. Takekawa, Acta Crystallogr. C 46, 1917 (1990).

${ }^{8}$ N. Ikeda, H. Ohsumi, K. Ohwada, K. Ishii, T. Inami, K. Kakurai, Y. Murakami, K. Yoshii, S. Mori, Y. Horibe, and H. Kito, Nature (London) 436, 1136 (2005).

${ }^{9}$ J. Iida, M. Tanaka, Y. Nakagawa, S. Funahashi, N. Kimizuka, and

S. Takekawa, J. Phys. Soc. Jpn. 62, 1723 (1993).

${ }^{10}$ A. D. Christianson, M. D. Lumsden, M. Angst, Z. Yamani, W. Tian, R. Jin, E. A. Payzant, S. E. Nagler, B. C. Sales, and D. Mandrus, Phys. Rev. Lett. 100, 107601 (2008).

${ }^{11}$ Y. Yamada, K. Kitsuda, S. Nohdo, and N. Ikeda, Phys. Rev. B 62, 12167 (2000).

${ }^{12}$ W. Wu, V. Kiryukhin, H.-J. Noh, K.-T. Ko, J.-H. Park, W. Ratcliff, P. A. Sharma, N. Harrison, Y. J. Choi, Y. Horibe, S. Lee, S. Park,
H. T. Yi, C. L. Zhang, and S.-W. Cheong, Phys. Rev. Lett. 101, 137203 (2008)

${ }^{13}$ F. Wang, J. Kim, Y. J. Kim, and G. D. Gu, Phys. Rev. B 80, 024419 (2009).

${ }^{14}$ J. P. Wright, J. P. Attfield, and P. G. Radaelli, Phys. Rev. Lett. 87, 266401 (2001).

${ }^{15}$ Y. Zhang, H. X. Yang, C. Ma, H. F. Tian, and J. Q. Li, Phys. Rev. Lett. 98, 247602 (2007).

${ }^{16}$ Y. Yamada, S. Nohdo, and N. Ikeda, J. Phys. Soc. Jpn. 66, 3733 (1997).

${ }^{17}$ H. J. Xiang and M.-H. Whangbo, Phys. Rev. Lett. 98, 246403 (2007).

${ }^{18}$ M. A. Subramanian, T. He, J. Chen, N. S. Rogado, T. G. Calvarese, and A. W. Sleight, Adv. Mater. 18, 1737 (2006).

${ }^{19}$ S. Funahashi, J. Akimitsu, K. Shiratori, N. Kimizuka, M. Tanaka, and H. Fujishita, J. Phys. Soc. Jpn. 53, 2688 (1984).

${ }^{20}$ N. Ikeda, R. Mori, S. Mori, and K. Kohn, Ferroelectrics 286, 175 (2003).

${ }^{21}$ S. Mori, S. Shinohara, Y. Matsuo, Y. Horibe, K. Yoshii, and N. Ikeda, Jpn. Jour. Appl. Phys. 47, 7595 (2008).

${ }^{22}$ M. Naka, A. Nagano, and S. Ishihara, Phys. Rev. B 77, 224441 (2008).

${ }^{23}$ Y. Horibe, N. Ikeda, K. Yoshii, and S. Mori, Phys. Rev. B 82, 184119 (2010). 
${ }^{24}$ Y. Nakawaga, M. Kishi, H. Hiroyoshi, N. Kimizuka, and K. Siratori, Ferrites, Proceedings of the Third International Conference on Ferrites, Kyoto (CAPJ, Tokyo, 1981), p. 115.

${ }^{25}$ J. Rodriguez-Carvajal, Physica B 192, 55 (1993).

${ }^{26}$ V. Petricek, M. Dusek, and L. Palatinus, Jana2006. The crystallographic computing system (Institute of Physics, Praha, Czech Republic, 2006).

${ }^{27}$ Y. Zhang, H. X. Yang, Y. Q. Guo, C. Ma, H. F. Tian, J. L. Luo, and J. Q. Li, Phys. Rev. B 76, 184105 (2007).

${ }^{28}$ M. H. Phan, N. A. Frey, M. Angst, J. de Groot, B. C. Sales, D. G. Mandrus, and H. Srikanth, Solid State Commun. 150, 341 (2010).

${ }^{29}$ J. Bourgeois, G. André, S. Petit, J. Robert, M. Poienar, J. Rouquette, E. Elkaïm, M. Hervieu, A. Maignan, C. Martin, and F. Damay, Phys. Rev. B (unpublished).

${ }^{30}$ C. Li, X. Zhang, Z. Cheng, and Y. Sun, Appl. Phys. Lett. 3, 152103 (2008).

${ }^{31}$ X. S. Xu, J. de Groot, Q. C. Sun, B. C. Sales, D. Mandrus, M. Angst, A. P. Litvinchuk, and J. L. Musfeldt, Phys. Rev. B 82, 014304 (2010).

${ }^{32}$ H. J. Noh, H. Sung, J. Jeong, J. Jeong, S. B. Kim, J. Y. Kim, J. Y. Kim, and B. K. Cho, Phys. Rev. B 82, 024423 (2010).

${ }^{33}$ M. Angst, R. P. Hermann, A. D. Christianson, M. D. Lumsden, C. Lee, M. H. Whangbo, J. W. Kim, P. J. Ryan, S. E. Nagler, W. Tian, R. Jin, B. C. Sales, and D. Mandrus, Phys. Rev. Lett. 101, 227601 (2008).

${ }^{34}$ M. I. Aroyo, J. M. Perez-Mato, C. Capillas, E. Kroumova, S. Ivantchev, G. Madariaga, A. Kirov, and H. Wondratschek, Zeitschrift für Kristallographie 221, 15 (2006).

${ }^{35}$ N. Ikeda, S. Mori, and K. Kohn, Ferroelectrics 314, 41 (2005).

${ }^{36}$ X. S. Xu, M. Angst, T. V. Brinzari, R. P. Hermann, J. L. Musfeldt, A. D. Christianson, D. Mandrus, B. C. Sales, S. McGill, J. W. Kim, and Z. Islam, Phys. Rev. Lett. 101, 227602 (2008).

${ }^{37}$ F. M. Vitucci, A. Nucara, D. Nicoletti, Y. Sun, C. H. Li, J. C. Soret, U. Schade, and P. Calvani, Phys. Rev. B 81, 195121 (2010).

${ }^{38}$ J. A. Tjon and M. Blume, Phys. Rev. 165, 456 (1968).

${ }^{39}$ M. Tanaka, K. Siratori, and N. Kimizuka, J. Phys. Soc. Jpn. 53, 760 (1984).

${ }^{40}$ A. M. Mulders, S. M. Lawrence, U. Staub, M. Garcia-Fernandez, V. Scagnoli, C. Mazzoli, E. Pomjakushina, K. Conder, and Y. Wang, Phys. Rev. Lett. 103, 077602 (2009).
${ }^{41}$ S. Park, Y. Horibe, Y. J. Choi, C. L. Zhang, S. W. Cheong, and W. Wu, Phys. Rev. B 79, 180401(R) (2009).

${ }^{42}$ S. Cao, J. Li, H. F. Tian, Y. B. Qin, L. J. Zeng, H. X. Yang, and J. Q. Li, App. Phys. Lett. 98, 102102 (2011).

${ }^{43}$ H. X. Yang, H. F. Tian,Y. Zhang, Y. B. Qin, L. J. Zeng, C. Ma, H. L. Shi, and J. B. Lu, Solid State Commun. 150, 1467 (2010).

${ }^{44}$ J. Bourgeois, A. M. Abakumov, F. Damay, C. Martin, and M. Hervieu (unpublished).

${ }^{45}$ Y. Matsuo, Y. Horibe, S. Mori, K. Yoshii, and N. Ikeda, J. Magn. Magn. Mater. 310, e349 (2007).

${ }^{46}$ A. M. Mulders, S. M. Lawrence, U. Staub, M. Garcia-Fernandez, V. Scagnoli, C. Mazzoli, E. Pomjakushina, K. Conders, and Y. Wang, Phys. Rev. Lett. 103, 077602 (2009).

${ }^{47}$ N. Tannieres, O. Evrard, and J. Aubry, C. R. Acad. Sci. C 278, 760 (1974).

${ }^{48}$ N. Kimizuka, A. Takenaka, Y. Asasada, and T. Katsura, Solid State Commun. 15, 1199 (1974).

${ }^{49}$ B. Malaman, O. Evrard, N. Tannieres, A. Courtois, and J. Protas, Acta Cryst. B 32, 749 (1976).

${ }^{50}$ A. J. Hearmon, D. Prabhakaran, H. Nowell, F. Fabrizi, M. J. Gutmann, and P. G. Radaelli, Phys. Rev. B 85, 014115 (2012).

${ }^{51}$ Y. Horibe, K. Yoshii, N. Ikeda, and S. Mori, Phys. Rev. B 80, 092104 (2009).

${ }^{52}$ K. Conder, Mater. Sci. Eng., R 32, 41 (2001).

${ }^{53}$ A. Nemudry, M. Weiss, I. Gainutdinov, V. Boldyrev, and R. Schönllhorn, Chem. Mater. 10, 2403 (1998).

${ }^{54}$ T. Sekine and T. Katsura, J. Solid State Chem. 17, 49 (1976).

${ }^{55}$ R. Gérardin, I. Nodari, H. Aqachmar, and O. Evrard, C. R. Acad. Sc. Paris 295, 863 (1982).

${ }^{56}$ I. Nodari, A. Alebouyeh, J. F. Brice, R. Gérardin, and O. Evrard, Mater. Res. Bull. 23, 1039 (1988).

${ }^{57}$ S. Patankar, S. K. Pandey, V. R. Reddy, A. Gupta, A. Banerjee, and P. Chaddah, EPL 90, 57007 (2010).

${ }^{58}$ M. Nespolo, M. Isobe, J. Iida, and N. Kimizuka, J. Alloys Compd. 313, 59 (2000).

${ }^{59}$ M. Nespolo, M. Isobe, and J. Iida, Acta Cryst. B 56, 805 (2000).

${ }^{60}$ M. Nespolo, M. Nakamura, and H. Ohashi, J. Solid State Chem. 150, 103 (2000).

${ }^{61}$ C. Gleitzer and J. B. Goodenough, Mixed-Valence Iron Oxides, Structure and Bonding (Springler-Verlag, Berlin Heidelberg, 1985), Vol. 61. 